\title{
A Novel Short-Range Prediction Model for Railway Track Irregularity
}

\author{
Peng Xu, Rengkui Liu, Quanxin Sun, and Futian Wang \\ MOE Key Laboratory for Urban Transportation Complex Systems Theory, Beijing Jiaotong University, \\ No. 3 Shang Yuan Cun, Haidian District, Beijing 100044, China \\ Correspondence should be addressed to Peng Xu, suepen_aili@126.com
}

Received 27 August 2012; Revised 12 November 2012; Accepted 15 November 2012

Academic Editor: Wuhong Wang

Copyright (C) 2012 Peng Xu et al. This is an open access article distributed under the Creative Commons Attribution License, which permits unrestricted use, distribution, and reproduction in any medium, provided the original work is properly cited.

\begin{abstract}
In recent years, with axle loads, train loads, transport volume, and travel speed constantly increasing and railway network steadily lengthening, shortcomings of current maintenance strategies are getting to be noticed from an economical and safety perspective. To overcome the shortcomings, permanent-of-way departments throughout the world have given a considerable attention to an ideal maintenance strategy which is to carry out appropriate maintenances just in time on track locations really requiring maintenance. This strategy is simplified as the conditionbased maintenance (CBM) which has attracted attentions of engineers of many industries in the recent 70 years. To implement CBM for track irregularity, there are many issues which need to be addressed. One of them focuses on predicting track irregularity of each day in a future short period. In this paper, based on track irregularity evolution characteristics, a Short-Range Prediction Model was developed to this aim and is abbreviated to TI-SRPM. Performance analysis results for TI-SRPM illustrate that track irregularity amplitude predictions on sampling points by TI-SRPM are very close to their measurements by Track Geometry Car.
\end{abstract}

\section{Introduction}

Traffic accidents have long been a social-economical problem which has caused increasing concerns to the public worldwide [1,2]. According to statistics on train accidents by Office of Safety of US Federal Railroad Administration, 542933 people were injured or killed by railway accidents mainly resulting from railway track from January 1975 to May 2011 [3].

The roles of railway track are to guide the vehicle or locomotive traveling, endure comprehensive effects of contributing environmental variables and loads from wheels, distribute wheel loads to roadbed, bridges, or tunnels, provide a smooth trajectory for wheel running, keep operation costs, travel safety and passenger comfort within an accepted range, and roadbed in a good condition $[4,5]$. Track condition may be classified into two categories: 
track geometry condition and track structure condition [6]. Track geometry condition plays a significant role in ensuring train traveling safety and passenger comfort [7] and is usually measured by several geometry parameters [5]. These parameters comprise projections of rails in the horizontal, longitudinal and vertical planes and include gauge and cross level in the horizontal plan, longitudinal level in the vertical plan, alignment in the longitudinal plane, and twist for measuring uneven running surface [8]. In theory, values of these five parameters that is, gauge, cross level, longitudinal level, alignment, and twist are $G_{0}$ which is the designed gauge and in most countries is equal to $1435 \mathrm{~mm}, 0 \mathrm{~mm}, 0 \mathrm{~mm}, 0 \mathrm{~mm}$, and $0 \mathrm{~mm} / \mathrm{m}$ for the straight track, respectively, and for the curved track are $G_{0}+G_{w}$, wherein $G_{w}$ is the designed gauge widening, and $C L_{0}$ which is the designed super-elevation, $0 \mathrm{~mm}$, $0 \mathrm{~mm}$ and $0 \mathrm{~mm} / \mathrm{m}$, respectively. Because of many reasons $[4,9,10]$, however, the actual values of these parameters deviate from their theoretical ones. These deviations are usually referred to as track irregularity [5]. The existence of track Irregularity makes unsmooth the running trajectory of wheels. The unsmooth trajectory will lead to vibrations of vehicles and locomotives and dynamic forces between wheels and rails. The trains vibrations and dynamic forces not only reduce train travelling safety and passenger comfort, but also shorten track equipment life $[4,11]$, what is more, deteriorating track irregularity further $[4,12]$. According to investigation reports, which were published by the Rail Accident Investigation Branch of UK, of 184 accidents occurred between October 2005 and June 2010, there are 58 derailments, and 24 of them are induced by bad track irregularity [13].

In recent years, with axle loads, train loads, transport volume, and travel speed constantly increasing and railway network steadily lengthening, shortcomings of current maintenance strategies are getting to be noticed from an economical and safety perspective [9]. To overcome the shortcomings, permanent-of-way departments throughout the world have given a considerable attention to an ideal maintenance strategy which is to carry out appropriate maintenances just in time on track locations requiring maintenance. This strategy is simplified as the condition-based maintenance (CBM) which has attracted attentions of engineers of many industries in the recent 70 years. To implement CBM strategy for track irregularity, there are many issues which need to be addressed [9]. One of them deals with predicting track irregularity of each day in the future short period.

There are some studies on track irregularity prediction methods. Professionals at Railway Technical Research Institute in Japan have, respectively, developed degradation models which utilize standard deviations of track geometry measurements to predict standard deviations of track alignment and surface over 100-meter-long track sections [14]. Alfelor et al. have built a track degradation database storing track gauge restraints and track geometry parameters measured with Gage Restraint Measurement System, traffic loads, environment, and track structural characteristics, and they developed a track degradation analysis program which can formulate a one-to-one relationship between track degradation and a contributing parameter by employing least square linear regression [15]. Chen et al. at China Academy of Railway Sciences have developed an integrated factor method (IFM) which utilizes the latest track geometry measurements to predict track irregularity parameter values on all sampling points [10]. In order to make predictions, IFM divides a railway track into 200-meter-long unit sections, classifies unit sections into 17 categories, and assumes that unit sections in the same category have the same track irregularity deterioration rate. Based on a common denominator; "a good track behaves well, while a poorer one deteriorates faster," Veit and Marschnig developed an exponential model for describing track irregularity evolution between two adjacent temping maintenances over a 5-meter-long track section [12]. Quiroga and Schnieder also developed an exponential model for describing track surface 
evolution between two adjacent temping maintenances over 200-meter-long sections [7]. Based on experiences of railway engineers, Meier-Hirmer et al. employed Gamma process to fit track surface deterioration rate between two adjacent temping maintenances over track sections with the length equal to 200 meters and 1000 meters [16]. Xu et al. developed a multistage framework integrating linear regression models to describe track irregularity evolution between two adjacent maintenances [17]. And based on conclusions drawn from $[9,16], \mathrm{Xu}$ et al. then proposed a novel method for predicting track irregularity condition over unit sections [18] and mean of track irregularity amplitudes over 10-meter-long unit sections [19].

There are some limitations of these methods [9]. The study presented in this paper will overcome these shortcomings and develop a novel, distinctive model to predict track irregularity parameter values over sampling points of each day within the following one or two inspection intervals of Track Geometry Car (TGC). Thus, the model is named ShortRange Prediction Model for Track Irregularity and is abbreviated to TI-SRPM.

The rest of the paper is organized as follows. Section 2 declares variables for TISRPM. In Section 3, TI-SRPM is detailed. In Section 4, by analyzing track gauge and surface predictions of about 8000 sampling points, the performance of TI-SRPM is analyzed. Based on the performance analysis results, conclusions are drawn in Section 5.

\section{Variable Denotations}

Variable denotations throughout this paper are listed as follows.

Sec: a track section on which TI-SRPM is built.

Ti: a track irregularity index for which TI-SRPM is built.

$\left\{t_{n, x}\right\}$ : a date series of every day within two adjacent maintenances at Sec; when $x$ is equal to $0, t_{n, 0}$ presents the date when a TGC inspection was carried out; when $x$ is more than $0, t_{n, x}$ presents the date of the $x$ th day after the $n$th TGC inspection; when $x$ is less than $0, t_{n, x}$ presents the date of the $x$ th day before the $n$th TGC inspection.

$a_{n, x}^{l}$ : the measurement by TGC of the amplitude of the track irregularity index Ti on $t_{n, x}$ over the sampling point $l$ in Sec; due to the fact that TGC is not operated to measure track irregularity each day, only $a_{n, 0}^{l}$ has the actual value.

$\hat{a}_{n, x}^{l}$ : the prediction of $a_{n, x}^{l}$.

$$
\begin{aligned}
A_{n} & =\left(\begin{array}{ccc}
a_{n, 0}^{1} & \cdots & a_{n, x}^{1} \\
\vdots & \ddots & \vdots \\
a_{n, 0}^{l} & \cdots & a_{n, x}^{l}
\end{array}\right), \\
\widehat{A}_{n} & =\left(\begin{array}{ccc}
\widehat{a}_{n, 1}^{1} & \cdots & \widehat{a}_{n, x}^{1} \\
\vdots & \ddots & \vdots \\
\hat{a}_{n, 1}^{l} & \cdots & \widehat{a}_{n, x}^{l}
\end{array}\right),
\end{aligned}
$$




$$
\begin{aligned}
& A_{n, x}=\left[a_{n, x}^{1}, \ldots, a_{n, x}^{l}\right]^{T}, \\
& \widehat{A}_{n, x}=\left[\widehat{a_{n, x}^{1}}, \ldots, \widehat{a}_{n, x}^{l}\right]^{T}, \\
& A_{n}^{l}=\left[a_{n, 1}^{l} \ldots a_{n, x}^{l}\right], \\
& \widehat{A}_{n}^{l}=\left[\widehat{a}_{n, 1}^{l} \ldots \widehat{a}_{n, x}^{l}\right] .
\end{aligned}
$$

$S_{n}$ : the condition of Ti over Sec on $t_{n, 0}$.

$k_{n}$ : the degradation rate of Ti within a date interval including the date of the $n$th TGC inspection.

$V_{l}=[\underbrace{1,1, \ldots, 1}_{l}]^{T}$, a column vector with the size of $l$ and all elements of 1.

\section{TI-SRPM}

Many factors exert influences on track irregularity deteriorations. To ensure TI-SRPM to capture track irregularity degradation trend as much as possible, it should be built on the basis of track irregularity degradation characteristics.

\subsection{TI-SRPM Research Object}

According to practical experiences on track irregularity degradation, under the effect of many factors on track irregularity deterioration, degradation processes of two near spatial points are different, and what is more, degradation processes of two track irregularity indices over a spatial point are also distinguished. On the other hand, maintenance works routinely or temporarily scheduled will destroy the current degradation process and initiate a new evolution process. Therefore, track irregularity prediction model should be built for the degradation process of a track irregularity index over a spatial point within two adjacent maintenance works.

However, because there are mileage errors existing between TGC inspections $[9,20$, $21]$, building track irregularity prediction model on a spatial point is usually impossible. Therefore, some researches on track irregularity predictions built models for a section of some length.

Track Geometry Measurements (TGMs) used in this paper are processed with two mileage error correction models, Key Equipment-based Mileage Error Correction model, KEBMEC [9, 22], and Track Geometry Measurements-based Mileage Error Correction model, TGM-BMEC [9]. Mileage errors of such TGM processed by these two models are normally less than a sampling distance, that is, $0.25 \mathrm{~m}$. Therefore, TI-SRPM will be constructed for the degradation process of a track irregularity index Ti over a shorter unit section, $0.5 \mathrm{~m}$. Due to the fact that a unit section only has $2 \sim 3$ sampling points, amplitudes of Ti over these points do not deviate very much from each other. Therefore, track irregularity condition, $S_{n}$, of Ti over Sec on $t_{n, 0}$ is quantified with the mean of track irregularity measurements of Ti over sampling points in Sec. 


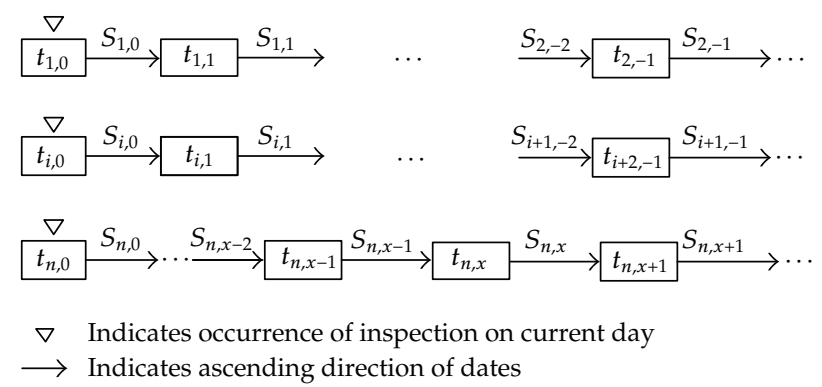

Figure 1: Evolutionary process of track irregularity over the unit section Sec.

\subsection{Track Irregularity Degradation Process of Ti over the Unit Section Sec}

With the current track irregularity condition as its initial condition, future track irregularity condition is induced by comprehensive influences of many factors on track irregularity. During railway transportation, track irregularity changes on a daily basis. The difference in track irregularity condition on two moments quantifies accumulatively comprehensive effects in the period. It indicates that comprehensive influences of factors on track irregularity deteriorations can be taken into consideration by directly using TGM. This forms a basis for some pieces of research on track irregularity predictions, for example, IFM in [9].

Therefore, $s_{n, x-1}$ on $t_{n, x-1}$ is a main input affecting $s_{n, x}$ on $t_{n, x}$. Meanwhile, $s_{n, x}$ serves as a main output at $t_{n, x}$ which influences $s_{n, x+1}$ on $t_{n, x+1}$. Figure 1 illustrates this evolution process. In the evolution process of Figure 1, track irregularity condition is inspected by TGC at an inspection cycle. Track irregularity condition $s_{n, x}$ on the day of $t_{n}$ can be calculated from TGM generated during this day's inspection.

\subsection{TI-SRPM Building}

Research results of [8] show that in a normal circumstance (no natural disasters occurring) and with no maintenance works carried out, track irregularity condition changes over a unit section within a short period are small. On the other hand, under effects of factors, changes in track irregularity condition over a unit section are nonlinear with railway transportation $[7,9-11,14,16-19]$. So, in the normal circumstance, the track irregularity degradation process can be presented as an approximately differentiable and evolutionarily smooth curve. According to one of calculus fundamentals, a nonlinear deterioration process in a short-range period within two adjacent maintenance works can be approximated by a linear deterioration process in the same short period. A whole evolution process between two adjacent maintenance works comprises deterioration processes of many short periods. Therefore, the whole process can be approximated by many linear deterioration processes.

According to the previous discussion, once the short period lengths are known for the approximation of a nonlinear deterioration process over Sec by many linear processes, TGM can then be used to fit these linear deterioration processes, and a mean deterioration rate, $k_{q}$, is available for each of these linear processes. $k_{q}$ quantifies the mean comprehensive effect of many factors on the condition of Ti over Sec. Because the unit section has a shorter length, all sampling points in Sec can be considered to have the same deterioration rate, $k_{q}$, for the track 


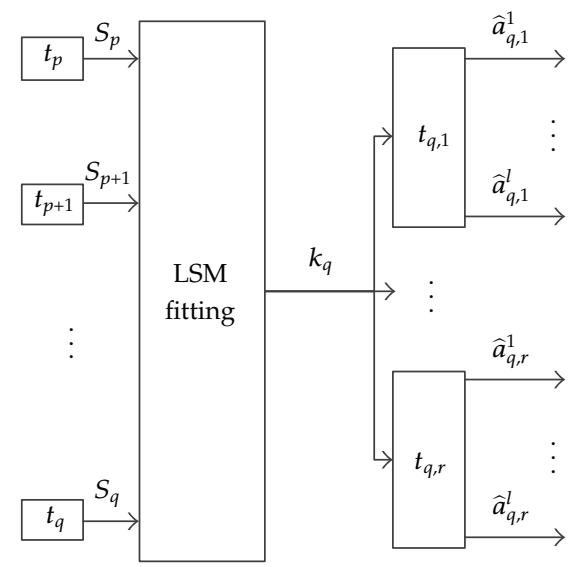

Figure 2: Illustration of predicting track irregularity amplitudes over all sampling points in the unit section Sec after the $q$ th Track Geometry Car inspection.

irregularity index Ti. According to the analysis results of Section 3.2, the amplitude of Ti over a sampling point $l$ on $t_{q, x}$ is approximated by $a_{q, 0}^{l}+k_{q} \cdot x$.

After the $q$ th TGC inspection for the railway track where the unit section Sec is, predicting amplitudes of Ti at each day within the period of $t_{q, 1} \sim t_{q, r}$ over sampling points in $\mathrm{Sec}$ is illustrated in Figure 2. In Figure 2, the date range satisfies the following two conditions: (a) $t_{q, r}-t_{p}=T_{q}$, wherein $T_{q}$ represents the short period length for the approximation of the current nonlinear deterioration process, and (b) in the period of $t_{q, 1} \sim t_{q, r}$, there is at least one TGC inspection for the railway track where Sec is. $r$ is determined by the TGC inspection interval and usually takes on the maximum interval, $I_{d}$. In Figure 2, the prediction for amplitudes $A_{q, x}$ of Ti on $t_{q, x}$ over sampling points in Sec is made with (3.1), wherein the mean deterioration rate, $k_{q}$, is determined by fitting the time series $\left\{S_{p}, S_{p+1}, \ldots, S_{q}\right\}$ with the least squares method, where

$$
\widehat{A}_{q, x}=A_{q, 0}+k_{q} \cdot x \cdot V_{l}
$$

Because the track irregularity deterioration is nonlinear, to approximate the real deterioration process with a linear process, when a new TGC inspection for the railway track is carried out, the newly generated TGM should be taken into consideration to adjust previously fitted deterioration rate, $k_{q}$, and to obtain a new one, $k_{q+1}$. The new deterioration rate is then used for making predictions as shown in Figure 3. In Figure 3, the date range satisfies the same conditions with one in Figure 2. Predictions for amplitudes, $A_{q+1, x}$, of Ti on $t_{q, x+1}$ in Sec are made according to (3.2). The mean deterioration rate in (3.2) is obtained according to the newly formed time series $\left\{S_{u}, S_{u+1}, \ldots, S_{q+1}\right\}$ where

$$
\widehat{A}_{q+1, x}=A_{q+1,0}+k_{q+1} \cdot x \cdot V_{l}
$$




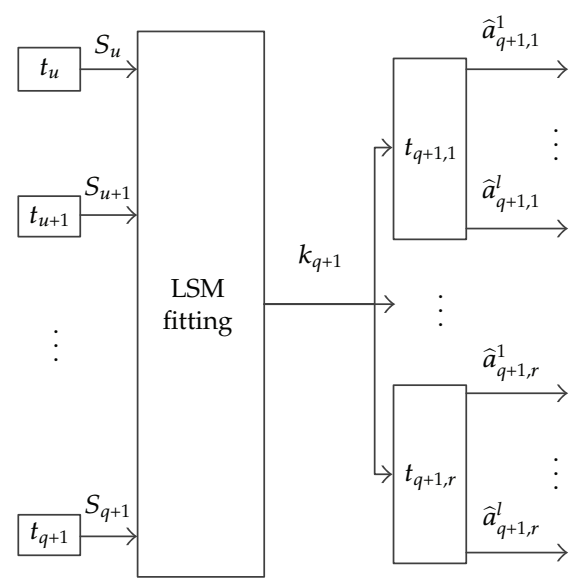

Figure 3: Illustration for predicting track irregularities of all sampling points along the unit section Sec after the $(q+1)$ th Track Geometry Car inspection.

As the TGC inspection for the railway track where Sec is continues, the previous adjustments will list a family of linear prediction models, shown in (3.3). The family of linear models is TI-SRPM for making short-range predictions for amplitudes of Ti over sampling points in Sec consider

$$
\begin{gathered}
\widehat{A}_{2, x}=A_{2,0}+k_{2} \cdot x \cdot V_{l} \\
\vdots \\
\widehat{A}_{q, x}=A_{q, 0}+k_{q} \cdot x \cdot V_{l}
\end{gathered}
$$

In (3.3), $x$ lies in the closed section $[1, r]$, and the mean deterioration rate, $k_{q}$, for each linear model is determined with the least squares methods by fitting $\left\{S_{p}, S_{p+1}, \ldots, S_{q}\right\}$ calculated from TGM generated during the least 4 TGC inspections. When the available TGC inspections are less than 4 in times, all available TGM are used.

\section{Performance Analysis}

Utilizing TGM processed with both KE-BMEC and TGM-BMEC, multiple predictions for amplitudes of track irregularity over sampling points on the Jiulong-Beijing Railway Track under the jurisdiction of Jinan Railway Bureau have been made with TI-SRPM. In this section, errors in predictions of amplitudes of gauge and right surface over sampling points in the section from K612+000 to K614+000 will be analyzed. There are about $4000(2000 / 0.5)$ unit sections and about $8000(2000 / 0.25)$ sampling points in the 2-kilometer-long track section. To perform these predictions, approximately $48000(4000 * 6 * 2)$ linear models in the form of (3.1) or (3.2) are built automatically with a computer routine coded for TI-SRPM. 
Table 1: Some error statistics from gauge predictions of all sampling points in Jiulong-Beijing track section from K612+000 to K614+000.

\begin{tabular}{lccc}
\hline Date & & Error statistic & $\rho$ \\
\hline Sep. 24 & ME $(\mathrm{mm})$ & 0.1912 & 0.9884 \\
Oct. 10 & -0.0018 & 0.0660 & 0.9985 \\
Oct. 30 & -0.0019 & 0.3007 & 0.9690 \\
Nov. 13 & -0.0013 & 0.1062 & 0.9960 \\
Dec. 12 & -0.0022 & 0.1481 & 0.9921 \\
Dec. 25 & -0.0027 & 0.1030 & 0.9961 \\
\hline
\end{tabular}

These predictions of 6 times were made after inspections of September 8, September 24, October 10, October 30, November 13, and December 12, 2008, respectively. As stated in Section 3, due to the fact that TGC does not perform inspections every day, track irregularity measurements are only available for those days with inspection. Thus, error analysis is carried out only for these predictions which have amplitude measurements, that is, on September 24, October 10, October 30, November 13, December 12, and December 25, 2008.

\subsection{Gauge}

Histograms of errors in gauge predictions are illustrated in Figure 4. And some statistics from gauge prediction errors such as Mean Error, ME, Standard Deviation, SD, and Correlation Coefficient, $\rho$, of errors are listed in Table 1.

Histograms in Figure 4 and statistics listed in Table 1 show that gauge prediction errors of these 6 times are normally distributed with means approximately equal to $0 \mathrm{~mm}$, the maximum SD is $0.3007 \mathrm{~mm}$, and the minimum $\rho$ takes the value of 0.9690 .

From the previous results, the following four main conclusions are arrived at: (a) the gauge deterioration trends over sampling points are captured by TI-SRPM, (b) gauge amplitude measurements, $a_{n, x}^{l}$, lie in the closed section [ $\hat{a}_{n, x}^{l}-2 \mathrm{SD}, \hat{a}_{n, x}^{l}+2 \mathrm{SD}$ ] (wherein, $\mathrm{SD}<0.31 \mathrm{~mm}$ ) with the probability of $95.44 \%$, (c) gauge predictions are very close to gauge measurements, and (d) there are high similarities between gauge measurements and predictions.

\subsection{Right Surface}

Figure 5 shows histograms of right surface prediction errors. Some error statistics are listed in Table 2.

Histograms in Figure 5 and error statistics listed in Table 2 show that right surface prediction errors are normally distributed with means approximately equal to $0 \mathrm{~mm}$, the maximum SD is $0.1487 \mathrm{~mm}$, and the minimum $\rho$ takes 0.9940 .

From the previous results, the similar conclusions with ones in Section 4.1 are drawn as follows: (a) right surface deterioration trends are captured by TI-SRPM, (b) right surface measurements, $a_{n, x}^{l}$, lie in the closed section $\left[\hat{a}_{n, x}^{l}-2 \mathrm{SD}, \hat{a}_{n, x}^{l}+2 \mathrm{SD}\right.$ ] (wherein, SD $<0.15 \mathrm{~mm}$ ) with the probability of $95.44 \%$, (c) right surface predictions are very close to right surface measurements, and (d) there are high similarities between right surface measurements and predictions. 


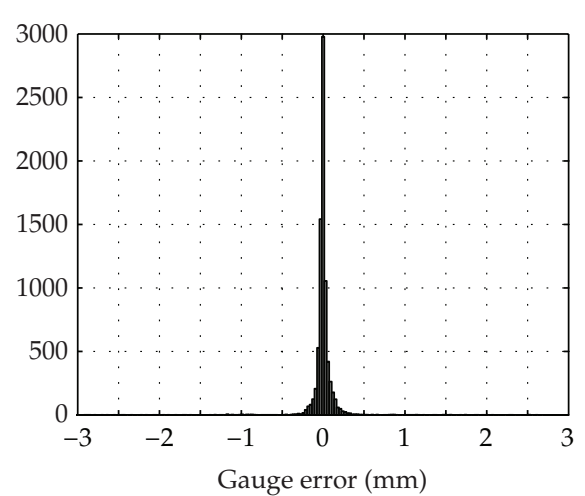

(a) September 24th

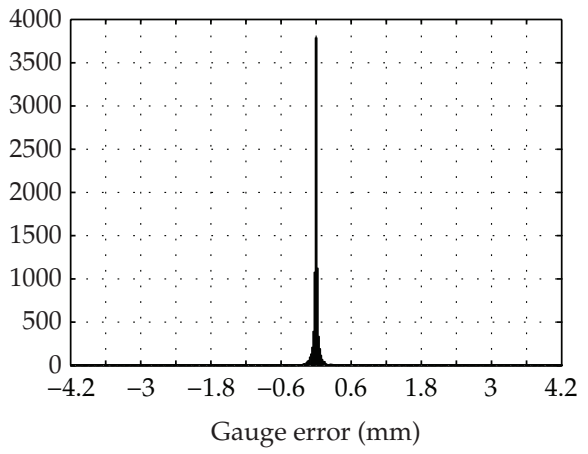

(c) October 30th

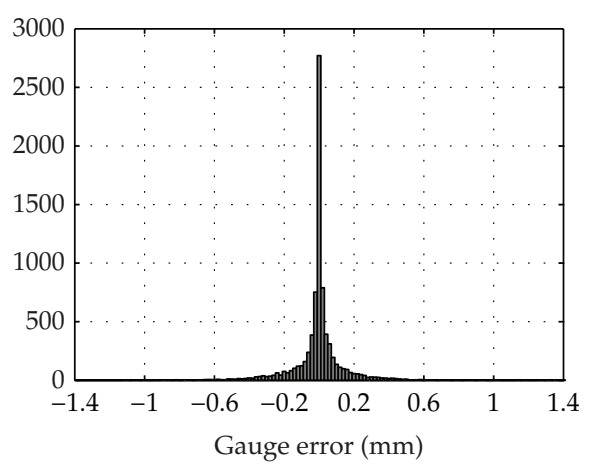

(e) December 12th

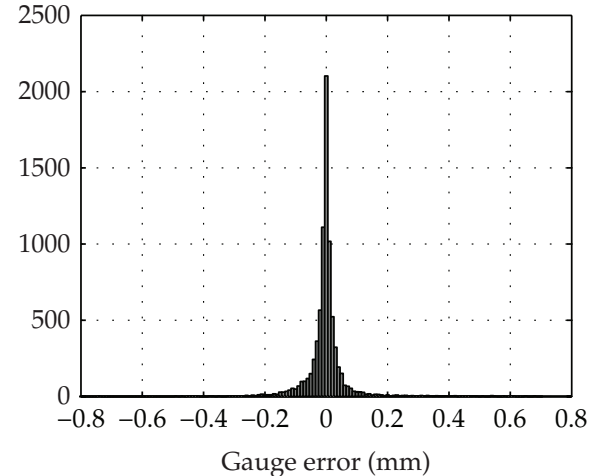

(b) October 10th

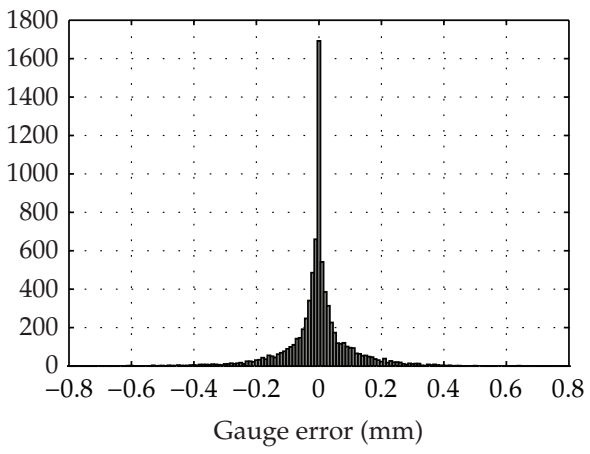

(d) November 13th

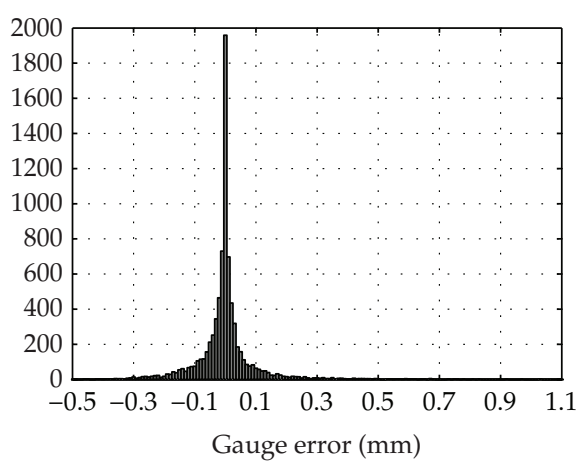

(f) December 25th

Figure 4: Histograms on errors in gauge predictions of all sampling points in Jiulong-Beijing track section from $\mathrm{K} 612+000$ to $\mathrm{K} 614+000$.

\section{Conclusions}

According to one of calculus fundamentals, on the basis of track irregularity deterioration characteristics, a short-range prediction model was developed for amplitudes of track irregularity over sampling points on each day within a future short period. The research objects of the model are a deterioration process of a track irregularity parameter between two adjacent maintenance works over a 0.5-meter-long unit section. TI-SRPM for a research 


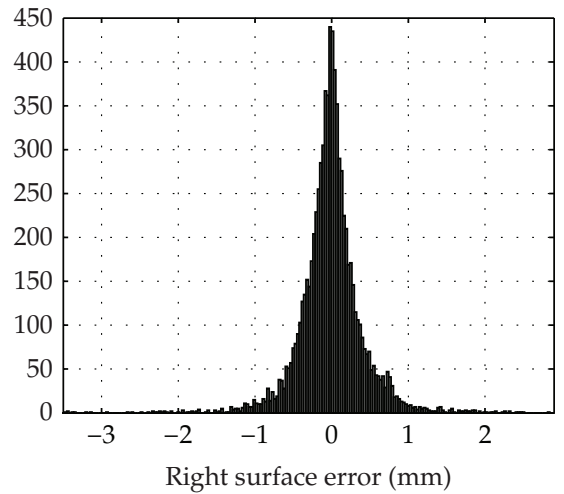

(a) September 24 th

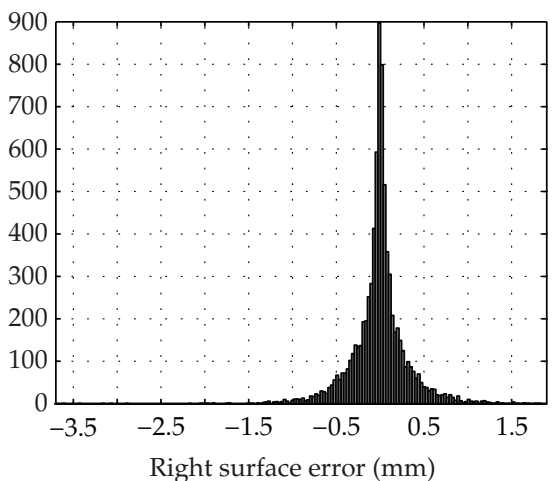

(c) October 30th

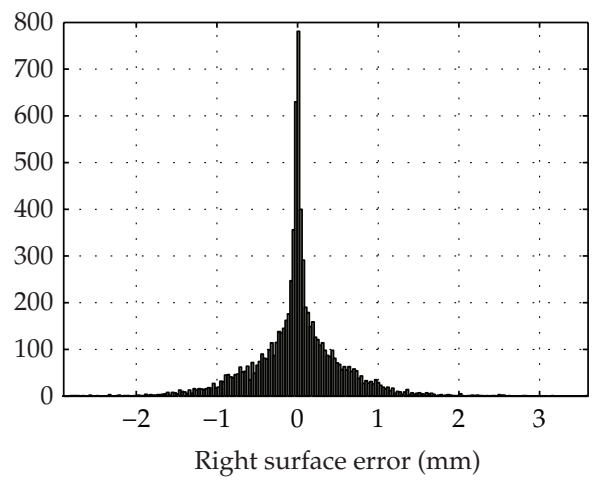

(e) December 12th

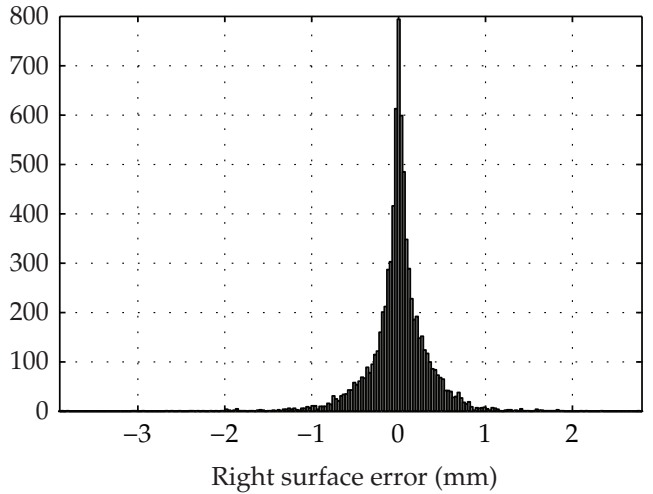

(b) October 10th

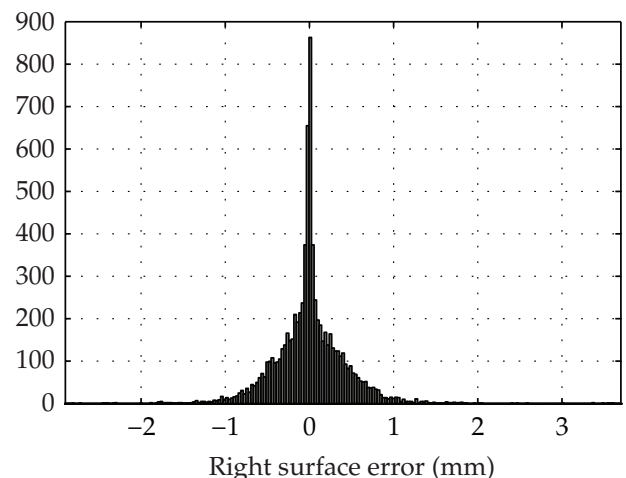

(d) November 13th

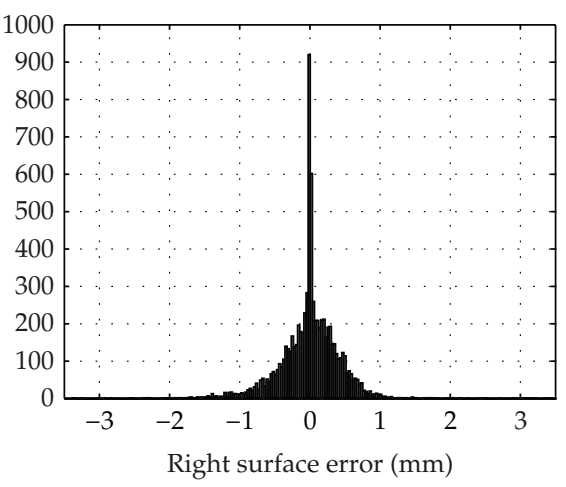

(f) December 25th

Figure 5: Histograms on errors in right surface predictions of all sampling points in Jiulong-Beijing track section from K612+000 to K614+000.

object comprises a family of linear models, that is, $\widehat{A}_{q, x}=A_{q, 0}+k_{q} \cdot x \cdot V_{l}$ (wherein, $q \geq 2$ and $x \in[1, r]$ ). Performance analysis in Section 4 for TI-SRPM shows that track irregularity deterioration trends over sampling points are captured by TI-SRPM, and track irregularity predictions are very close to their measurements. 
Table 2: Some error statistics from right surface predictions of all sampling points in Jiulong-Beijing track section from $\mathrm{K} 612+000$ to $\mathrm{K} 614+000$.

\begin{tabular}{lccc}
\hline Date & & Error statistic & $\rho$ \\
\hline Sep. 24 & ME $(\mathrm{mm})$ & SD $(\mathrm{mm})$ & 0.9940 \\
Oct. 10 & -0.0047 & 0.1477 & 0.9977 \\
Oct. 30 & -0.0015 & 0.0931 & 0.9975 \\
Nov. 13 & -0.0010 & 0.0976 & 0.9949 \\
Dec. 12 & 0.0019 & 0.1378 & 0.9943 \\
Dec. 25 & -0.0034 & 0.1486 & 0.9986 \\
\hline
\end{tabular}

\section{Acknowledgments}

This research was partly sponsored by the National Key Technology R\&D Program (2009BAG12A10) and the National Basic Research Program of China (2012CB725406).

\section{References}

[1] H. W. Guo, W. H. Wang, W. W. Guo, X. B. Jiang, and B. Heiner, "Reliability analysis of pedestrian safety crossing in urban traffic environment," Safety Science, vol. 50, no. 4, pp. 968-973, 2012.

[2] W. H. Wang, Vehicle's Man-Machine Interaction Safety and Driver Assistance, Communications Press, Beijing, China, 2012.

[3] Federal Railroad Administration Office of Safety Analysis-Train Accidents, http://safetydata.fra.dot .gov/OfficeofSafety/publicsite/Query/inctally1.aspx.

[4] S. Yoshihiko, New Railway Track Dynamics, China Railway Press, Beijing, China, 2001.

[5] C. Esveld, Modern Railway Track, Delft University of Technology Publishing Services, Delft, The Netherlands, 2nd edition, 2001.

[6] J. M. Sadeghi and H. Askarinejad, "Development of track condition assessment model based on visual inspection," Structure and Infrastructure Engineering, vol. 7, no. 12, pp. 895-905, 2011.

[7] L. M. Quiroga and E. Schnieder, "A heuristic approach to railway track maintenance scheduling," in Proceedings of the 12th International Conference on Computer System Design and Operation in the Railways and other Transit Systems (COMPRAIL '10), pp. 687-699, WIT Press, Beijing, China, September 2010.

[8] CEN, prEN13848-1:2003 Railway Applications-Track-Track Geometry Quality-Part 1: Characterization of Track Geometry, CEN, Brussels, Belgium, 2004.

[9] P. Xu, Mileage error correction model for track geometry data from track geometry car $\mathcal{E}$ track irregularity prediction model [dissertation], Beijing Jiaotong University, Beijing, China, 2012.

[10] X. M. Chen, L. Wang, F. C. Yang, X. S. Chai, and W. Q. Wu, "Integrating factor method for predicting the developing trend of railway track irregularity," China Railway Science, vol. 27, no. 6, pp. 27-31, 2006 (Chinese).

[11] J. J. Fan, Modern Railway Track, China Railway Press, Beijing, China, 2nd edition, 2004.

[12] P. Veit and S. Marschnig, "Sustainability in track-a precondition for high speed traffic," in Proceedings of ASME Joint Rail Conference (JRC'10), pp. 349-355, Urbana, Ill, USA, April 2010.

[13] Rail Accident Investigation Branch, Reports and bulletins, http://www.raib.gov.uk/publications/ investigation_reports.cfm.

[14] A. Kawaguchi, M. Miwa, and K. Terada, "Actual data analysis of alignment irregularity growth and its prediction model," Quarterly Report of RTRI, vol. 46, no. 4, pp. 262-268, 2005.

[15] R. M. Alfelor, G. A. Carr, and M. Fateh, "Track degradation assessment using gage restraint measurements," Transportation Research Record, no. 1742, pp. 68-77, 2001.

[16] C. Meier-Hirmer, G. Riboulet, F. Sourget, and M. Roussignol, "Maintenance optimization for a system with a gamma deterioration process and intervention delay: application to track maintenance," Journal of Risk and Reliability, vol. 223, no. 3, pp. 189-198, 2009. 
[17] P. Xu, R. K. Liu, F. T. Wang, Q. X. Sun, and H. L. Teng, "A novel description method for track irregularity evolution," International Journal of Computational Intelligence Systems, vol. 4, no. 6, pp. 13581366, 2012.

[18] P. Xu, Q. X. Sun, R. K. Liu, and F. T. Wang, "A short-range prediction model for track quality index," Journal of Rail and Rapid Transit, vol. 225, no. 3, pp. 277-285, 2011.

[19] R. Liu, P. Xu, and F. Wang, "Research on a short-range prediction model for track irregularity over small track lengths," Journal of Transportation Engineering, vol. 136, no. 12, pp. 1085-1091, 2010.

[20] E. T. Selig, G. M. Cardillo, E. Stephens, and A. Smith, "Analyzing and forecasting railway data using linear data analysis," in Proceedings of the 11th International Conference on Computer System Design and Operation in Railways and other Transit Systems, Toledo, Spain, September 2008.

[21] D. K. O'Brien, Optimal estimation and rail tracking analysis [dissertation], University of Massachusetts Lowell, Lowell, Mass, USA, 2005.

[22] P. Xu, Q. X. Sun, R. K. Liu, and F. T. Wang, "Key equipment identification model for correcting milepost errors of track geometry data from track inspection car," Transportation Research C. In press. 


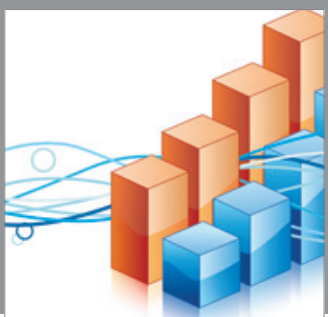

Advances in

Operations Research

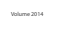

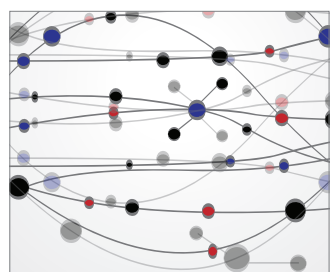

\section{The Scientific} World Journal
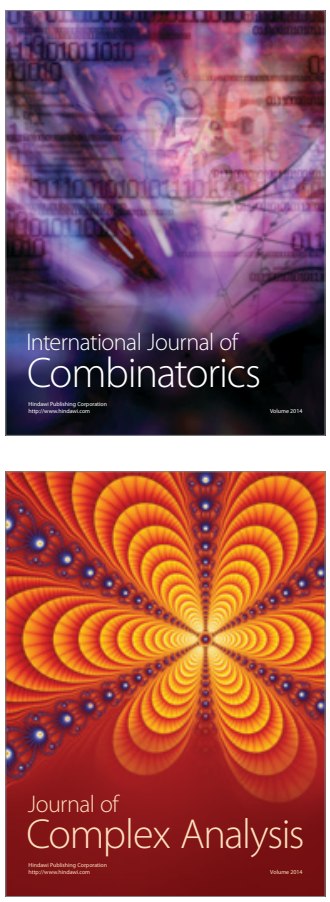

International Journal of

Mathematics and

Mathematical

Sciences
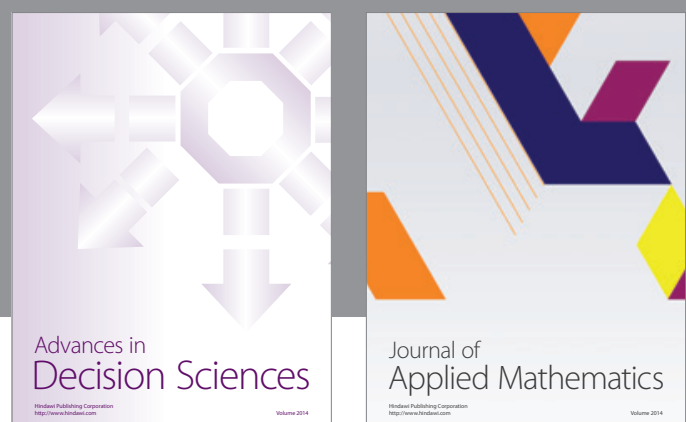

Journal of

Applied Mathematics
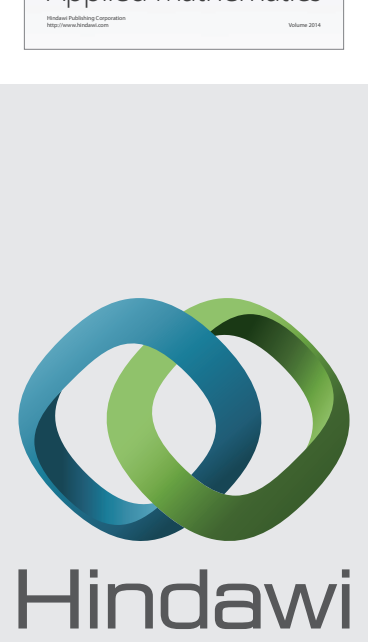

Submit your manuscripts at http://www.hindawi.com
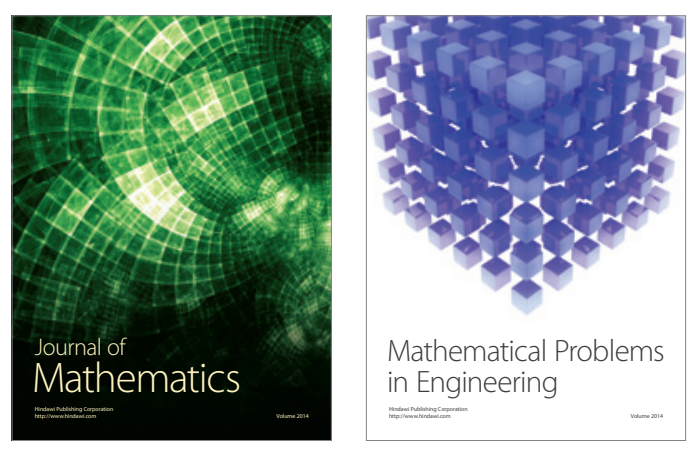

Mathematical Problems in Engineering
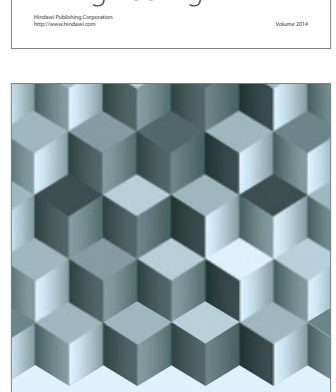

Journal of

Function Spaces
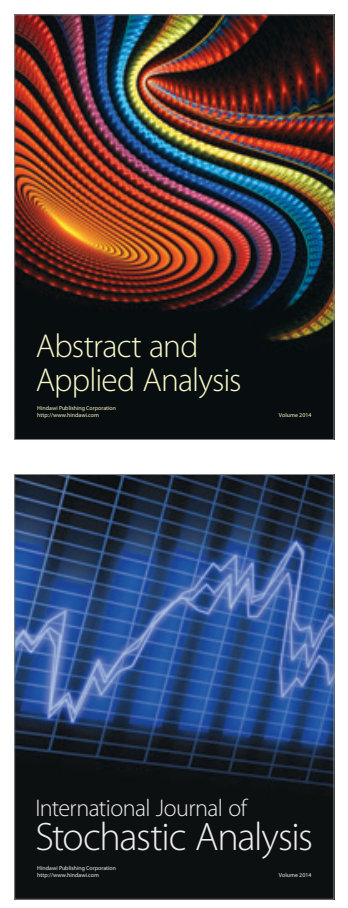

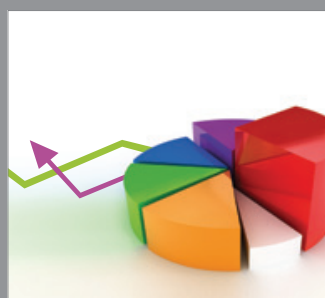

ournal of

Probability and Statistics

Promensencen
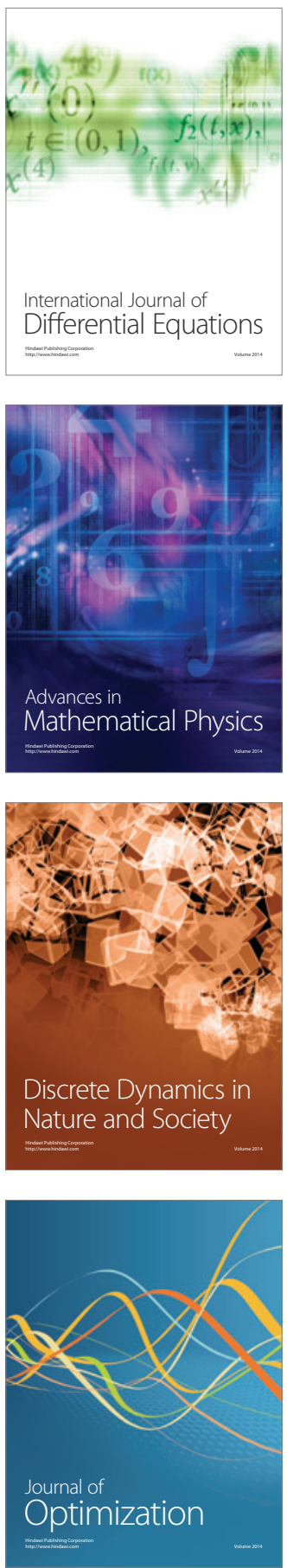